\title{
NMDA Di-Heteromeric Receptor Populations and Associated Proteins in Rat Hippocampus
}

\author{
Rana A. Al-Hallaq, ${ }^{1}$ Thomas P. Conrads, ${ }^{2}$ Timothy D. Veenstra, ${ }^{2}$ and Robert J. Wenthold ${ }^{1}$ \\ ${ }^{1}$ Laboratory of Neurochemistry, National Institute on Deafness and Other Communication Disorders, National Institutes of Health, Bethesda, Maryland \\ 20892, and 2Laboratory of Proteomics and Analytical Technologies, Science Applications International Corporation-Frederick, National Cancer Institute- \\ Frederick, National Institutes of Health, Frederick, Maryland 21702
}

Subunit composition of NMDA receptors (NMDARs) determines a range of physiological properties, downstream signaling effects, and binding partners. Differential localization of NR2A- or NR2B-containing NMDARs within the neuron and subunit-specific protein associations may explain differences in NR2A and NR2B contributions to synaptic plasticity and excitotoxic cell death. This question is complicated by the existence of tri-heteromeric complexes (NR1/NR2A/NR2B). To date, no quantitative biochemical determinations have been made of the relative abundance of different NMDAR populations in intact hippocampus, the region extensively correlated with NMDAR-dependent long-term potentiation. We investigated subunit composition and subunit-specific interactions in CA1/CA2 of rat hippocampus. Using sequential immunoprecipitations to deplete either NR2B or NR2A, di-heteromeric NR1/NR2A and NR1/NR2B receptor populations were isolated from postnatal day 7 (P7) hippocampus and P42 and 6-month-old CA1/CA2. Quantitative Western blot analysis revealed that $60-70 \%$ of NR2A and $70-85 \%$ of NR2B subunits were associated in NR1/NR2A or NR1/NR2B di-heteromeric complexes. Isolated di-heteromeric receptor fractions were used to examine NR2A- or NR2B-specific interactions with synapseassociated proteins. Our results indicate that NR2A- or NR2B-containing NMDARs associate similarly with postsynaptic density-95 (PSD-95), synapse-associated protein 102, and PSD-93 at P42. However, NR2A-containing receptors coimmunoprecipitated a greater proportion of the synaptic proteins neuronal nitric oxide synthase, Homer, and $\beta$-catenin. Finally, mass spectrometry analysis of isolated di-heteromeric receptors identified a novel NMDAR interactor, collapsin response mediator protein 2, which preferentially associates with NR2B-containing di-heteromeric NMDARs. In summary, in rat hippocampus, NR2A and NR2B exist primarily in di-heteromeric complexes that interact similarly with PSD-95-related proteins but are associated with different protein complexes.

Key words: tri-heteromeric; MAGUK; PSD-95; CRMP2; detergent solubility; postsynaptic

\section{Introduction}

The NMDA receptor (NMDAR) is a glutamate-gated ion channel with specialized features that contribute to its role in long-term potentiation (LTP), the molecular model of learning and memory extensively characterized in the hippocampus (Lynch, 2004). Furthermore, the receptor is implicated in a variety of neurological and neurodegenerative conditions, including ischemic injury and Huntington's disease (Gardoni and Di Luca, 2006). Its role is thought to be determined, at least in part, by subunit-dependent

\footnotetext{
Received May 10, 2007; revised June 11, 2007; accepted June 22, 2007.

This work was supported by the National Institute on Deafness and Other Communication Disorders (R.A.A. and R.J.W.) and National Cancer Institute (NCI) (T.P.C. and T.D.V.) Intramural Programs of National Institutes of Health (NIH). We are grateful to David Lucas (NCl, NIH, Frederick, MD) for his contributions to the mass spectrometry data analysis and to Dr. Ronald Petralia for editorial comments. We thank Drs. Georg Kohr (Max Planck Institute for Medical Research, Heidelberg, Germany), Barry Wolfe (Georgetown University, Washington, DC), and Paul Worley (Johns Hopkins School of Medicine, Baltimore, MD) for providing antibodies.

Correspondence should be addressed to Dr. Rana A. Al-Hallaq, National Institute on Deafness and Other Communication Disorders/National Institutes of Health, 50 South Drive, Mail Stop Code 8027, Building 50, Room 4146, Bethesda, MD 20892-8027. E-mail: alhallaq@nided.nih.gov.

T. P. Conrads' present address: Department of Pharmacology, University of Pittsburgh Cancer Institute, Pittsburgh, PA 15213.

DOI:10.1523/JNEUROSCI.2155-07.2007

Copyright $\odot 2007$ Society for Neuroscience $\quad$ 0270-6474/07/278334-10\$15.00/0
}

localization within the neuron and subunit-specific protein associations (Cull-Candy and Leszkiewicz, 2004; Kohr, 2006).

The functional NMDAR is most likely a tetramer of two NR1 and two NR2 subunits (Cull-Candy et al., 2001; Furukawa et al., 2005). Numerous studies have reported that subunit composition is a major determinant of multiple NMDAR properties, including channel kinetics (McBain and Mayer, 1994), regulation by posttranslational modifications such as phosphorylation (Kennedy and Manzerra, 2001; Salter and Kalia, 2004), and intracellular protein associations (Wenthold et al., 2003). For example, the postsynaptic density-95 (PSD-95) family of membrane-associated guanylate kinases (MAGUKs), implicated in NMDAR trafficking to and stabilization on the cell surface (Roche et al., 2001; Lin et al., 2004; Prybylowski et al., 2005), may differentially associate with or regulate the NMDAR subunits NR2A and NR2B (Sans et al., 2000; Losi et al., 2003; Townsend et al., 2003; Prybylowski et al., 2005). Furthermore, it has been suggested that NMDARs are localized to different subcellular compartments based on subunit composition, i.e., in adult, NR2Acontaining receptors are enriched at the synapse, whereas extrasynaptic receptors are predominantly NR2B-containing complexes (Stocca and Vicini, 1998; Tovar and Westbrook, 1999; Kohr, 2006). More recent evidence indicates that, because of dif- 
ferences in the downstream signaling cascades coupled to NR2A and NR2B, subunit composition may determine NMDAR contributions to excitotoxic cell death in various disease models (Lynch and Guttmann, 2002; Vanhoutte and Bading, 2003). Thus, characterization of NMDAR subunit composition is a key step in understanding NMDAR function.

NMDAR subunit composition is dependent on subunit availability, which differs as a function of brain region and developmental age (Watanabe et al., 1993; Wenzel et al., 1997; Sans et al., 2000). In the hippocampus, NR2B expression is high in early development and decreases with age, whereas NR2A expression begins later in development and increases into adulthood. Furthermore, NMDARs may exist not only as di-heteromeric receptors (NR1/NR2X), but also as tri-heteromeric receptors (NR1/ NR2X/NR2Y). Although the existence of a tri-heteromeric NR1/ NR2A/NR2B receptor population has been reported by electrophysiological (Wafford et al., 1993; Kew et al., 1998; Tovar and Westbrook, 1999; Kohr, 2006) and biochemical (Sheng et al., 1994; Stephenson, 2001) methods, no quantitative biochemical investigations have been conducted in intact hippocampus.

We set out to determine subunit composition of NMDARs in hippocampus using serial immunoprecipitations (IPs). We isolated di-heteromeric receptor populations, determined their relative abundance, and examined their interactions with MAGUKs and other synapse-associated proteins. Finally, using these isolated fractions and mass spectrometry analysis, we identified a novel interactor, collapsin receptor mediator protein 2 (CRMP2), which preferentially interacts with the NR1/NR2B subpopulation of di-heteromeric receptors.

\section{Materials and Methods}

Antibodies. The anti-NR2A (T58) and anti-NR2B (T51) rabbit polyclonal antibodies have been characterized previously (Christie et al., 1999). The mouse monoclonal antibody to NR2A was a kind gift from Dr. Georg Kohr (Max Planck Institute for Medical Research, Heidelberg, Germany), and the anti-NR2B mouse monoclonal antibody was a generous gift from Dr. Barry Wolfe (Georgetown University, Washington, DC) (Wang et al., 1995). Mouse monoclonal antibodies to the following proteins were purchased from the following: NR1 (clone 54.1) from BD Biosciences PharMingen (San Jose, CA); PSD-95 (clone K28/43), synapse-associated protein 102 (SAP102) (clone N19/2), PSD-93 (clone N18/30), and SAP97 (clone K64/15) from NeuroMab (Davis, CA); neuronal nitric oxide synthase (nNOS) (clone 16), and $\beta$-catenin (clone 14) from BD Transduction Labs (San Jose, CA); and CRMP2 (clone C4G) from Immuno-Biological Laboratories (Gunma, Japan). The rabbit polyclonal antibody to pan-Homer was a kind gift from Dr. Paul Worley (Johns Hopkins School of Medicine, Baltimore, MD). Affinity-purified horseradish peroxidase-conjugated protein $\mathrm{A}$ and sheep anti-mouse secondary antibodies were purchased from GE Healthcare Bio-Sciences (Piscataway, NJ).

Preparation of membrane fractions. Postnatal day 7 (P7), P42, and 6-month-old male Sprague Dawley rat brains were harvested as follows: P7 whole hippocampus was dissected and frozen until solubilization; P42 brains were harvested and frozen; and 6-month-old frozen rat brains were purchased from Zivic Laboratories (Pittsburgh, PA). Frozen P42 and 6-month-old brains were sliced, and the CA1/CA2 was dissected. P42 and 6-month-old CA1/CA2 and P7 whole hippocampus were placed immediately in ice-cold solubilization buffer $(50 \mathrm{~mm}$ Tris- $\mathrm{HCl}, \mathrm{pH} 7.5$, with protease inhibitors). Tissue was homogenized with a Polytron homogenizer, and membranes were sedimented by centrifugation $\left(100,000 \times g, 30 \mathrm{~min}, 4^{\circ} \mathrm{C}\right)$ and solubilized in $1 \%$ deoxycholate (DOC) for $45 \mathrm{~min}$ at $37^{\circ} \mathrm{C}$. After solubilization, $1 \%$ Triton X-100 was added, and the preparations were centrifuged at $100,000 \times g$ for $30 \mathrm{~min}$ to remove insoluble material. Protein concentrations were measured using the BCA Protein Assay kit (Pierce, Rockford, IL). Samples were stored at $-80^{\circ} \mathrm{C}$ or loaded onto agarose beads for immunoprecipitations.
Immunoprecipitations. Immunoprecipitation experiments were performed as described previously (Sans et al., 2000). Fifty microliters of protein A agarose beads (Pierce) were washed in wash buffer $(50 \mathrm{~mm}$ Tris- $\mathrm{HCl}, \mathrm{pH} 7.5$, and $0.1 \%$ Triton X-100). Ten microliters of normal rabbit serum, NR2A (T58) antiserum, or NR2B (T51) antiserum were coupled to the beads for $4 \mathrm{~h}$ or overnight at $4^{\circ} \mathrm{C}$ with constant rotation. After three washes with wash buffer, $500 \mathrm{mg}$ of total protein of P7, P42, or 6-month-old hippocampal or CA1/CA2 membrane fractions were incubated overnight at $4^{\circ} \mathrm{C}$ with the coupled antibody with constant rotation. For double immunoprecipitations, the unbound fraction was loaded onto an identical set of antibody-conjugated protein A beads for $6 \mathrm{~h}$ at $4^{\circ} \mathrm{C}$ with constant rotation. For the triple immunoprecipitations, the unbound fractions from the second immunoprecipitations were incubated overnight at $4^{\circ} \mathrm{C}$ with constant rotation with antibody-conjugated protein A beads as follows: normal serum onto normal serum, NR2A immunoprecipitation onto anti-NR2B-conjugated beads, and NR2B immunoprecipitation onto anti-NR2A-conjugated beads. All immunoprecipitations were washed three times with wash buffer including protease inhibitors and $150 \mathrm{~mm} \mathrm{NaCl}$. Fifty microliters of $2 \times$ SDS sample buffer was added, and the samples were boiled for $3 \mathrm{~min}$ and loaded onto 4-20\% polyacrylamide gels for SDS-PAGE.

Western blotting and analysis. Standard Western blotting procedures were followed (Sans et al., 2000). Twenty-eight micrograms of protein were loaded in each lane. Membranes were washed and incubated with primary antibody using the following concentrations: NR1 (54.1), 1:1000; NR2A (2F6.3D5), 1:5000; and NR2B monoclonal, $1.0 \mu \mathrm{g} / \mathrm{ml}$. Immunoreactive bands were visualized with ECL Plus Western Blotting Detection System (GE Healthcare Bio-Sciences).

Films were scanned for densitometric analysis using a Molecular Dynamics (Sunnyvale, CA) densitometer. For quantification of the diheteromeric receptor populations, the unbound fractions from the second immunoprecipitations were measured. Dilutions of input (100, 75, 50,25 , and $10 \%$ ) were analyzed and plotted as a standard curve. Experiments in which the unbound levels from control immunoprecipitations were $<50 \%$ of input were excluded. The levels of unbound NR2A or NR2B subunits were determined from the standard curve.

To analyze the coimmunoprecipitations of associated proteins (MAGUKs, nNOS, Homer, $\beta$-catenin, and CRMP2), the bound fractions from the third immunoprecipitations were analyzed. The levels of associated protein were normalized to the levels of NR1 subunit coimmunoprecipitated with anti-NR2A or anti-NR2B to provide a measure of associated protein per NMDAR immunoprecipitated. All experiments were performed with tissue from three or more animals ( $n$ is noted for each experiment). Values are presented as mean \pm SEM.

Nanoflow reversed-phase liquid chromatography-tandem mass spectrometry. Coomassie blue-stained protein gel bands were digested as described previously (Wilm et al., 1996). Samples were desalted with C18 Zip Tips (Millipore, Bedford, MA) as per the protocols of the manufacturer before nanoflow reversed-phase liquid chromatography (nanoRPLC) coupled on-line with tandem mass spectrometry (MS/MS).

Chromatographic separations were conducted using a microcapillary column with an integrated electrospray ionization (ESI) emitter constructed by flame pulling a fine tip $(\sim 5-7 \mu \mathrm{m}$ orifice $)$ on a $75 \mu \mathrm{m}$ inner diameter $\times 360 \mu \mathrm{m}$ outer diameter $\times 10 \mathrm{~cm}$ long segment of fused silica (Polymicro Technologies, Phoenix, AZ). This integrated ESI column was slurry packed in-house with $5 \mu \mathrm{m}, 300 \AA$ pore size C- 18 stationary phase (Jupiter; Phenomenex, Torrance, CA). The integrated ESI column was connected via a stainless steel union to an Agilent Technologies (Palo Alto, CA) 1100 nanoflow LC system, coupled on-line to a linear ion-trap mass spectrometer (LIT-MS) (LTQ; Thermo Electron, San Jose, CA). After sample injection, a 20 min wash with $98 \%$ mobile phase A $(0.1 \%$ $\mathrm{HCOOH}$ in water) was applied, and peptides were eluted using a linear gradient of $2 \%$ mobile phase $\mathrm{B}\left(0.1 \% \mathrm{HCOOH}\right.$ in $\left.\mathrm{CH}_{3} \mathrm{CN}\right)$ to $42 \%$ mobile phase B over $40 \mathrm{~min}$ with a constant flow rate of $200 \mathrm{nl} / \mathrm{min}$. The column was washed for 15 min with $98 \%$ mobile phase B and reequilibrated with $98 \%$ mobile phase A before subsequent sample loading.

The integrated nanoRPLC ESI column was coupled on-line to an LIT-MS using the nanoelectrospray source of the manufacturer with an 


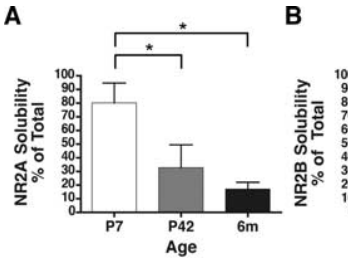

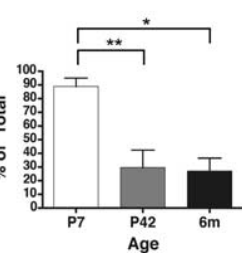
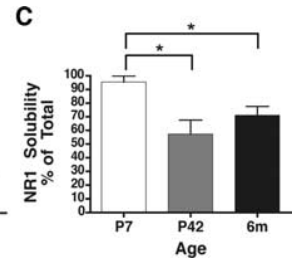

Figure 1. NR1, NR2A, and NR2B detergent solubility decreases with developmental age. Rat whole hippocampus (P7) or CA1/CA2 [P42 and 6 months $(6 \mathrm{~m})$ ] membrane fractions were solubilized with $1 \%$ DOC, resolved by SDS-PAGE, and subjected to densitometric analysis. The solubilized fraction is presented as a percentage of the original homogenate for NR2A $(A)$, NR2B $(B)$, and NR1 (C). NMDAR subunit solubility is significantly decreased at P42 and 6 months relative to $\mathrm{P7}$ for the subunits tested. Data are representative of three independent experiments performed on different hippocampal or CA1/CA2 preparations. Values are shown as mean \pm SEM. ${ }^{*} p<0.05 ;{ }^{* *} p<0.01$.

applied electrospray potential of $1.5 \mathrm{kV}$ and capillary temperature of $160^{\circ} \mathrm{C}$. The LIT-MS was operated in a data-dependent mode in which each full MS scan was followed by five MS/MS scans, in which the five most abundant peptide molecular ions detected from the MS scan were dynamically selected for five subsequent MS/MS scans using a collisional-induced dissociation (CID) energy of 35\%. Dynamic exclusion was used to minimize redundant MS/MS acquisition.

The CID spectra were analyzed using SEQUEST operating on a 40 node Beowulf parallel virtual machine cluster computer (Thermo Electron) and the Homo sapiens proteome database (http://www.expasy.org). Only peptides with conventional tryptic termini (allowing for up to two internal missed cleavages) possessing delta-correlation scores $\left[\left(\Delta C_{n}\right)>\right.$ 0.08 and charge state-dependent cross-correlation $\left(X_{\text {corr }}\right)$ criteria], as follows, were considered as legitimate identifications.

Statistical analysis. Data were analyzed in GraphPad Software (San Diego, CA) Prism 4.0. Significant differences between two groups were determined with a two-tailed, paired Student's $t$ test. Significant differences among multiple groups were determined with an ANOVA, followed by the Newman-Keuls multiple-comparison test. Differences were considered significant at $p<0.05$. All values are reported as mean \pm SEM, and $n$ indicates number of membrane fractions analyzed.

\section{Results \\ Detergent solubility of NMDAR subunits decreases with developmental age}

The NMDAR is only partially soluble in $1 \%$ DOC (Blahos and Wenthold, 1996), likely attributable to tight association of the receptor complex with the cytoskeleton, the protein-dense structure of the PSD, and/or lipid rafts (Allison et al., 1998; Hering et al., 2003; Besshoh et al., 2005). Approximately $35 \%$ of total NR2A and NR2B protein is extracted from adult rat forebrain membrane fractions by DOC (Blahos and Wenthold, 1996). Immunoprecipitation analysis requires a soluble protein fraction; thus, solubilization of NMDARs may determine the subpopulation of NMDARs that is investigated. Therefore, as an initial step, we examined the levels of NR1, NR2A, and NR2B in the DOCsolubilized fraction, which was used subsequently for immunoprecipitation experiments. P42 or 6-month-old CA1/CA2 of adult hippocampus was dissected, providing a relatively homogenous population of cells. Alternatively, P7 whole hippocampus was dissected. The levels of solubilized protein were measured relative to serial dilutions of the original tissue homogenate before solubilization, which represents the total NMDAR population.

At P42, the percentages of NR2A, NR2B, and NR1 solubility were $33 \pm 17,30 \pm 13$, and $57 \pm 10$ (mean \pm SEM; $n=3$ ), respectively (Fig. $1 A-C$ ), within the previously published ranges of solubility (Blahos and Wenthold, 1996). In early postnatal development, surface NMDARs are primarily extrasynaptic

(Washbourne et al., 2004), and the PSD is not fully developed (Petralia et al., 2005). These results suggest that the NMDAR may not be as tightly associated with the synapse in younger animals; therefore, we investigated NMDAR solubility from whole hippocampus at P7. As seen in Figure 1, $A$ and $B$, NR2A and NR2B subunit solubility was nearly threefold higher than in P42 CA1/ CA2 ( $p<0.05$ and $p<0.01$ between P7 and P42 for NR2A and $\mathrm{NR} 2 \mathrm{~B}$, respectively). In CA1/CA2 of 6-month-old animals, the solubility of NR2A and NR2B was similar to that seen for P42 (Fig. 1A,B) but differed significantly from solubility at P7 ( $p<$ 0.05 for NR2A and NR2B). Thus, NR2A and NR2B solubility is highest at P7 and decreases in older animals, corresponding to maturation of the PSD and/or increased NMDAR localization to the synapse.

In contrast, NR1 solubility was relatively high across developmental ages (Fig. $1 C$ ), perhaps reflecting the relatively large population of unassociated NR1 subunits (Chazot and Stephenson, 1997; Huh and Wenthold, 1999). Nevertheless, NR1 subunit solubility was nearly twofold higher at P7 than in P42 CA1/CA2. The solubility of NR1 at P7 differed significantly from P42 or 6 -month-old animals $(p<0.05)$. These findings suggest that, as with the NR2A and NR2B subunits, detergent solubility of NR1 decreases with age, perhaps attributable to increased synaptic localization or to tighter association of the NMDAR complex within mature synapses.

\section{Di-heteromeric NMDARs are isolated from P42 CA1/CA2 by serial immunoprecipitations}

NR1, NR2A, and NR2B are the predominant NMDAR subunits in the hippocampus based on evidence that NR2A and NR2B are the NR2 subunits most highly expressed in the hippocampus (Wenzel et al., 1997) and that NR3A and NR3B are present in a relatively small percentage of NMDARs (Al-Hallaq et al., 2002). Furthermore, previous studies have reported relatively low levels of unassembled NR2 subunits in cerebellar granule cells and cortical neurons (Luo et al., 1997; Huh and Wenthold, 1999); therefore, the majority of NR2A and NR2B subunits are presumably associated into either di-heteromeric (NR1/NR2A or NR1/ $\mathrm{NR} 2 \mathrm{~B}$ ) or tri-heteromeric (NR1/NR2A/NR2B) receptors.

Figure $2 \mathrm{~A}$ outlines the experimental plan used to isolate diheteromeric NMDARs from membrane fractions. P42 CA1/CA2 membrane fractions were subjected to two sequential immunoprecipitations with anti-NR2A or anti-NR2B antibodies to ensure a relatively complete clearing of the intended subunit contained in either the di-heteromeric or tri-heteromeric receptor population. This resulted in isolation of NR2B- or NR2Acontaining di-heteromeric receptors, respectively, represented by the IP II Unbound fraction. In subsequent experiments, these isolated populations were subjected to a third round of immunoprecipitation, generating the IP III Bound fraction, to determine the composition of the di-heteromeric receptor complexes.

We first verified the efficiency of the polyclonal anti-NR2A and anti-NR2B antibodies for immunoprecipitation. Membrane fractions from $\mathrm{P} 42$ rat hippocampus were subjected to immunoprecipitation analysis. The polyclonal anti-NR2A antibody immunoprecipitated NR2A (Fig. 2B, IP I Bound, lane 2), and a corresponding decrease in signal was seen in the unbound fraction (IP I Unbound, lane 2). Similarly, when the anti-NR2B antibody was used to immunoprecipitate the hippocampal membrane fractions, NR2B was detected in the IP I Bound fraction (Fig. $2 C$, lane 3), with a corresponding decrease in signal in the unbound fraction (IP I Unbound, lane 3). Furthermore, NR2B was coimmunoprecipitated with NR2A (Fig. 2C, IP I Bound, lane 
A

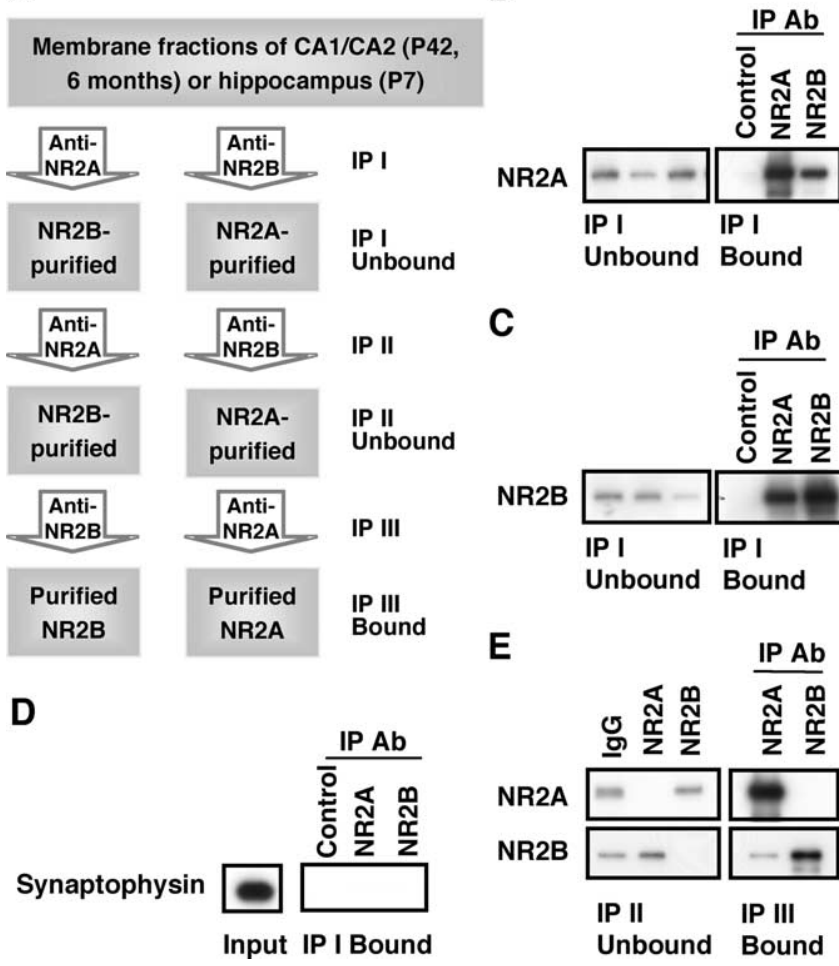

Figure 2. NR2A and NR2B di-heteromeric receptor populations are isolated by serial immunoprecipitations. A, A flowchart of the experimental procedure outlining the serial immunoprecipitations used to examine di-heteromeric receptors and their associated proteins. The IP II Unbound fraction corresponds to the NR2A-or NR2B-associated di-heteromeric populations. $\boldsymbol{B}$, C, P42 hippocampal membrane fractions were immunoprecipitated by normal rabbit serum, anti-NR2A, or anti-NR2B antibodies (Ab), and the IP I Unbound and IP I Bound fractions were immunoblotted with antibodies to NR2A $(\boldsymbol{B})$ or NR2B $(\boldsymbol{C})$. The anti-NR2A and -NR2B antibodies efficiently immunoprecipitate their respective antigen as well as smaller amounts of the other subunit. $\boldsymbol{D}$, In similar experiments, NR2A- or NR2B-bound fractions were subjected to immunoblotting with an antibody to synaptophysin. A strong signal was detected in the input, but no signal was detected in the IP I Bound fractions, suggesting that the NR2A and NR2B immunoprecipitations are specific for an NMDAR protein complex. $\boldsymbol{E}$, Representative blots after double and triple immunoprecipitations with normal rabbit serum, anti-NR2A, or anti-NR2B antibodies. The IP II Unbound fractions verify that the NR2A-depleted (lane 2) or NR2B-depleted (lane 3) fractions do not contain detectable level of NR2A or NR2B, respectively. The IP III Bound fractions indicate that the NR2B-depleted fraction (lane 1) contains NR2A but a low level of NR2B, and the NR2A-depleted fraction (lane 2) contains NR2B but no detectable levels of NR2A, confirming efficient isolation of NR2A- or NR2B-containing di-heteromeric receptor populations. For all experiments, $14 \mu$ l of the input, unbound, and bound fractions were resolved by SDS-PAGE and probed. Because the bound fractions were resuspended in $0.1 \mathrm{vol}$ of the original input, the bound sample is 10 times more concentrated than the input or unbound fractions.

2), and NR2A was coimmunoprecipitated with NR2B (Fig. 2B, IP I Bound, lane 3), consistent with several reports showing that NR2A and NR2B coexist within the same NMDAR complexes (Blahos and Wenthold, 1996; Kew et al., 1998). The normal rabbit serum immunoprecipitations provided a measure of protein loss during washes. The presynaptic protein synaptophysin did not coimmunoprecipitate with NR2A or NR2B (Fig. 2D), confirming that the NMDAR antibodies specifically immunoprecipitated an NMDAR complex.

Thus, NR2A and NR2B may be immunoprecipitated efficiently from P42 CA1/CA2. We next set out to isolate diheteromeric NMDARs by double-immunoprecipitation experiments. The IP II Unbound fraction was subjected to SDS-PAGE, and a representative blot is show in Figure $2 E$. In samples immunoprecipitated with anti-NR2A, no detectable NR2A remained in the unbound fraction (IP II Unbound, top, lane 2), confirming an efficient removal of NR2A subunits. Similarly, in samples subjected to double immunoprecipitation with the anti-NR2B antibody, no signal was detected for NR2B in the unbound fraction (IP II Unbound, bottom, lane 3).

Next, to determine the purity of the remaining di-heteromeric receptor populations (NR1/NR2A or NR1/NR2B), we immunoprecipitated the NR2B- or NR2A-depleted fractions with antiNR2A or anti-NR2B antibodies, respectively (Fig. 2 E, right, IP III Bound fraction). The NR2B-depleted fraction was immunoprecipitated with anti-NR2A, and a robust signal was detected for NR2A (top, lane 1). Despite the depletion of NR2B by serial immunoprecipitations with an anti-NR2B antibody, NR2B was also detected after anti-NR2A immunoprecipitation (IP III Bound, bottom, lane 1). However, we estimate this residual signal to be $\leq 5 \%$ of the original input based on the following evidence. First, our threshold for signal detection was $\leq 5 \%$ based on serial dilutions of the input fraction (data not shown), suggesting that lack of detectable signal in the IP II Unbound fraction (Fig. 2 E, IP II Unbound, lane 2 for NR2A and lane 3 for NR2B) represents no more than $5 \%$ of the original input. Second, because the bound fractions are 10 times as concentrated as the unbound fractions and because the signal for NR2B that coimmunoprecipitated with the NR2A-isolated fraction was no greater than half of that in the input (Fig. 2E, IP III Bound, bottom, lane 1, compared with IP II Unbound, bottom, lane 1), we calculate that $\leq 5 \%$ of NR2B remained in the NR2B-depleted fraction.

Conversely, immunoprecipitation of the NR2B from the isolated NR2B-containing di-heteromeric receptor population resulted in a robust signal for NR2B (Fig. 2E, IP III Bound, bottom, lane 2). When the NR2A-depleted fraction was immunoprecipitated with anti-NR2B, no NR2A was detected in the bound fraction (top, lane 2), suggesting that no residual NR2A remained. Thus, we estimate that double immunoprecipitations result in the removal of at least $95 \%$ of the intended subunit and in the isolation of the di-heteromeric receptor populations.

\section{Isolation of di-heteromeric receptor populations indicates that NR2A and NR2B are primarily in complexes of NR1/NR2A or NR1/NR2B}

To investigate the relative abundance of the di-heteromeric NMDAR populations, NR2A and NR2B remaining after double immunoprecipitations were measured by densitometric analysis and quantified relative to a series of dilutions of the original input (representing total NMDARs). A representative blot of the unbound fraction after double immunoprecipitations from $\mathrm{P} 42$ CA1/CA2 is shown in Figure 3A. NR2A or NR2B were efficiently depleted, resulting in the isolated NR1/NR2B or NR1/NR2A diheteromeric receptor populations, respectively. Quantification of the unbound fraction revealed that the percentage of NR2A unbound after double immunoprecipitations with anti-NR2B, representing the NR1/NR2A di-heteromeric receptor population, was $59 \pm 19 \%$ of the original input (mean \pm SEM; $n=3$ ) (Fig. 3D). Similarly, double immunoprecipitation with anti$\mathrm{NR} 2 \mathrm{~A}$, resulting in an isolated NR1/NR2B population, revealed that $71 \pm 15 \%$ of NR2B was present in this di-heteromeric receptor population (mean \pm SEM) (Fig. $3 D$ ). Presumably, the remaining 41 and $29 \%$ of NR2A and NR2B, respectively, that were bound to each other constitute NR1/NR2A/NR2B triheteromeric receptors. Thus, NR1/NR2A, NR1/NR2B, and NR1/ NR2A/NR2B complexes in hippocampus each constitute approximately one-third of the total NMDAR population. 
A

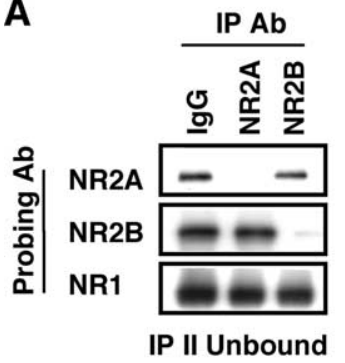

C

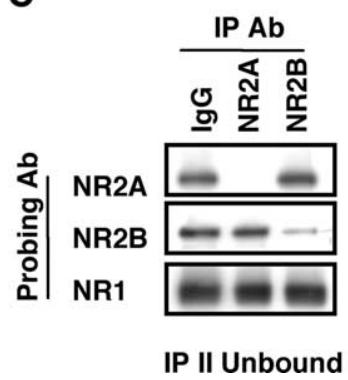

B

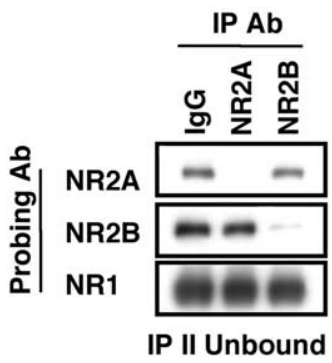

D

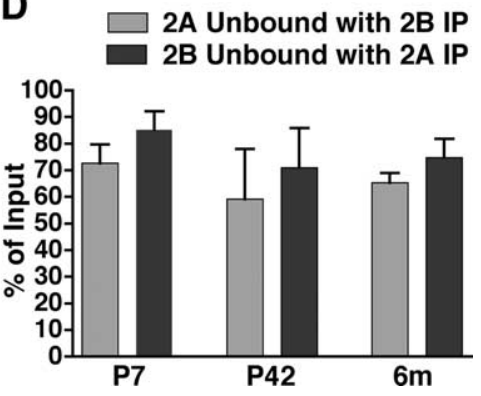

Figure 3. NR2A and NR2B are in di-heteromeric NR1/NR2A or NR1/NR2B or in triheteromeric NR1/NR2A/NR2B complexes throughout development. P42 CA1/CA2 $(\boldsymbol{A}, \boldsymbol{D}), \mathrm{P} 7$ hippocampus $(\boldsymbol{B}, \boldsymbol{D})$, and 6-month-old $C A 1 / C A 2(\boldsymbol{C}, \boldsymbol{D})$ were subjected to double immunoprecipitations with anti-NR2A or anti-NR2B antibodies $(A b)$. $A-C$, Representative immunoblots of the IP II Unbound fractions probed with anti-NR2A, -NR2B, or -NR1 antibodies demonstrating that the majority of NR2A (lane 2) or NR2B (lane 3) has been depleted (compare with normal rabbit serum, lane 1). D, Densitometric analysis of P7 hippocampus, P42 CA1/CA2, and 6-month-old $(6 \mathrm{~m})$ CA1/CA2 of the unbound fraction normalized to the input. Data are representative of three independent experiments performed on different hippocampal or CA1/CA2 preparations. Values are shown as mean \pm SEM.

The composition of NMDAR complexes is dependent on the availability of NMDAR subunits, the expression of which is regulated over development (Watanabe et al., 1993; Wenzel et al., 1997; Sans et al., 2000). We used these variations in the NR2A to NR2B ratio, which increases with age, to investigate whether the size of the di-heteromeric receptor populations differed as a result of relative subunit availability. Following the same protocol as that used to determine the P42 di-heteromeric receptor population (Fig. $3 A, D$ ), we examined the levels of di-heteromeric NMDARs in P7 hippocampus (Fig. $3 B, D$ ) and 6-month-old CA1/CA2 (Fig. 3C,D).

At P7, NR2A- or NR2B-containing di-heteromeric receptors were isolated at an efficiency similar to that seen at P42 (Fig. 3B). Quantification of the unbound fraction relative to original input resulted in $73 \pm 7$ and $85 \pm 7 \%$ of NR2A and NR2B remaining after double immunoprecipitations with anti-NR2B or antiNR2A antibodies, respectively (Fig. 3D). Similarly, at 6 months, the di-heteromeric receptor populations were isolated (Fig. $3 C$ ), and the sizes of the di-heteromeric receptor population were similar to those seen at P7 and P42 (Fig. 3D). Thus, despite the differences in subunit expression over developmental age, 60$70 \%$ of NR2A and $70-85 \%$ of NR2B subunits were associated with NR1/NR2A or NR1/NR2B di-heteromeric complexes at the ages examined.

\section{NR1/NR2A and NR1/NR2B receptors coimmunoprecipitate similarly with MAGUKs}

Because the di-heteromeric receptor populations constitute a substantial portion of the NMDARs in hippocampus and because the double immunoprecipitations allow for the isolation of these fractions, we set out to determine the proteins associated with these populations. The MAGUKs are a family of PDZ (PSD-95/ Discs large/zona occludens-1) domain-containing proteins that serve a scaffolding function in the synapse and possible roles in trafficking of the NMDAR (Wenthold et al., 2003). Previous studies have suggested a preferential interaction of PSD-95 with NR2A and of SAP102 with NR2B, based on the developmental expression patterns of these proteins and coimmunoprecipitation studies (Sans et al., 2000; Townsend et al., 2003). To investigate the existence of NR2A- or NR2B-preferential interactions with PSD-95 or SAP102, respectively, we examined associations of the MAGUKs with the isolated di-heteromeric receptor fractions.

After double immunoprecipitations from P42 CA1/CA2, the IP II Unbound fraction (NR1/NR2A and NR1/NR2B) was subjected to a third round of immunoprecipitations with anti-NR2A or anti-NR2B antibodies, generating the IP III Bound fraction (Fig. $2 A$ ). In Figure $4 A$, representative blots are shown of the IP II Unbound (left column) and the IP III Bound (right column) fractions. The samples were immunoblotted with anti-PSD-95, -SAP102, -PSD-93, -SAP97, and -NR1 antibodies. As seen in the lanes containing the bound fractions, the levels of PSD-95 that were coimmunoprecipitated with NR2A and NR2B were similar. After quantitative analysis, in which the levels of PSD-95 coimmunoprecipitated with NR2A or NR2B were normalized to the amount of NMDAR immunoprecipitated (as measured by NR1 coimmunoprecipitation), there was no significant difference between PSD-95 coimmunoprecipitated with NR2A and NR2B $(n=8 ; p=0.085)$ (Fig. $4 B)$.

Similarly, when SAP102 and PSD-93 were examined, no visible difference was detected in NR2A- and NR2B-associated fractions (Fig. $4 A$ ). When the coimmunoprecipitates were subjected to quantitative analysis, no significant differences were measured in the associations with NR2A and NR2B for SAP102 $(n=8 ; p=$ 0.162 ) (Fig. $4 B$ ) or PSD-93 $(n=4 ; p=0.085)$ (Fig. $4 B)$. Although SAP97 was present in the input fractions (Fig. $4 A$ ), no SAP97 was detected in the immunoprecipitations with NR2A or NR2B, consistent with findings from previously published studies (Sans et al., 2000) (but see Gardoni et al., 2003). Thus, the di-heteromeric NR1/NR2A and NR1/NR2B populations interact to similar levels with PSD-95, SAP102, and PSD-93 in the hippocampus.

Di-heteromeric NMDARs predominate at all ages examined in this study (Fig. 3); however, synaptic constituents differ over developmental age (Petralia et al., 2005). Thus, we examined the association of MAGUK proteins with NR2A- and NR2Bcontaining di-heteromeric receptor populations in P7 hippocampal membrane fractions. The signal, however, was below our threshold for detection (data not shown) and, therefore, could not be quantified. We confirmed the interaction of NMDARs with the MAGUKs at P7 from whole hippocampus after a single immunoprecipitation with anti-NR2A or antiNR2B antibodies (data not shown). PSD-95, SAP102, and PSD-93 coimmunoprecipitated with NR2A- and NR2Bcontaining receptors. Similar to our findings in P42 CA1/CA2 (Fig. 4A), SAP97 was not detected after single immunoprecipitations with anti-NR2A and -NR2B antibodies. Thus, members of the MAGUK family of proteins interacted similarly with the general population of NR2A and NR2B in P7 hippocampus, but associations with the di-heteromeric receptors were below detectable levels. 


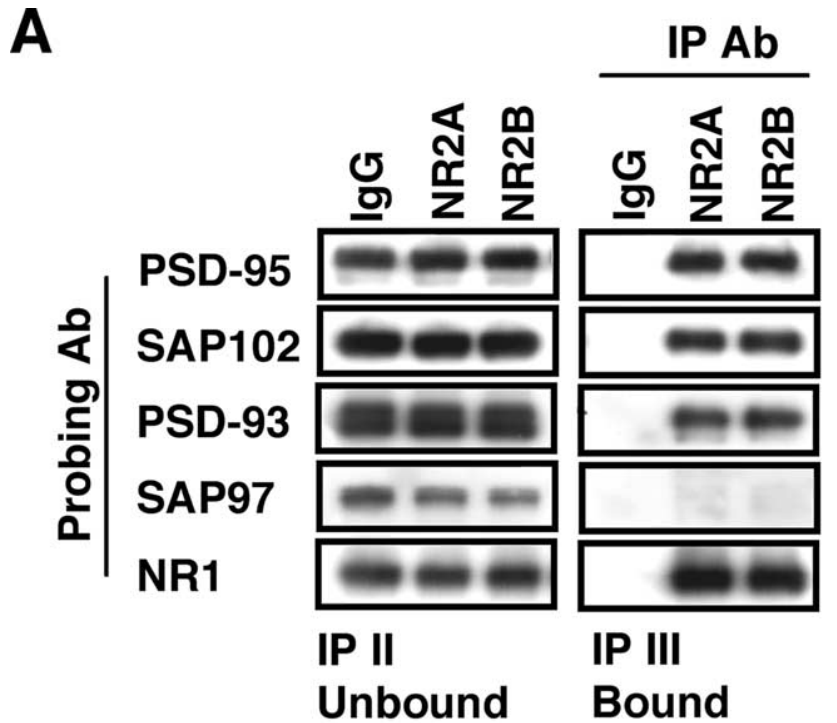

B

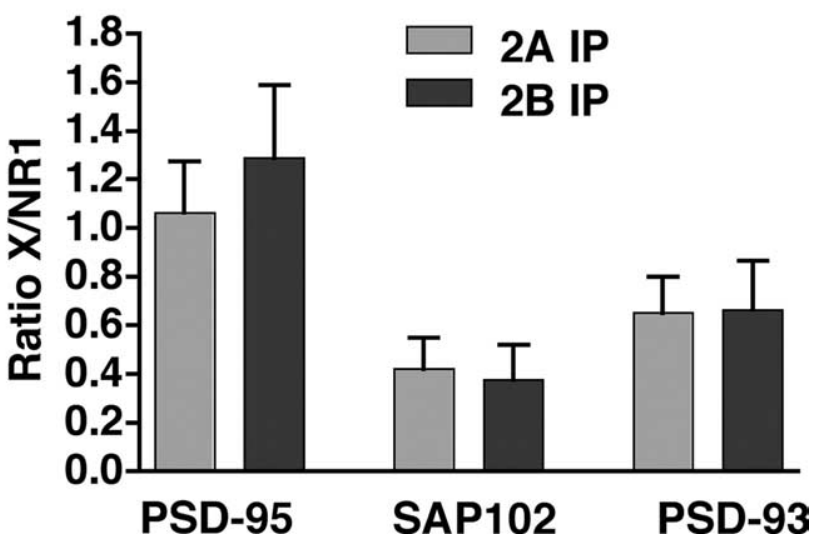

Figure 4. PSD-95, SAP102, and PSD-93 associate to a similar extent with the NR1/NR2A or NR1/NR2B di-heteromeric receptors in P42 CA1/CA2. Immunoprecipitations with anti-NR2A and anti-NR2B antibodies from isolated di-heteromeric receptor fractions (NR1/NR2A and NR1/ NR2B) were analyzed for coassociation with the PSD-95 family of proteins. $\boldsymbol{A}$, Representative immunoblots of the IP II Unbound and IP III Bound fractions after triple immunoprecipitations and immunoblotting with anti-PSD-95, -SAP102, -PSD-93, -SAP97, and -NR1 antibodies (Ab). With the exception of SAP97, NR2A- and NR2B-bound fractions coimmunoprecipitate similar levels of the proteins investigated. $\boldsymbol{B}$, Densitometric analysis of the levels of PSD-95, SAP102, or PSD-93 coimmunoprecipitated with NR2A- and NR2B-containing di-heteromeric receptors. All data are normalized to the level of NMDAR immunoprecipitated, as measured by NR1 coimmunoprecipitations, and no significant differences are found between NR2A- or NR2Bimmunoprecipitated fractions for any of the proteins. Data are representative of eight (PSD-95 and SAP102) or four (PSD-93) independent experiments performed on different CA1/CA2 preparations. Values are shown as mean \pm SEM.

\section{NR1/NR2A and NR1/NR2B-containing receptors} coimmunoprecipitate differentially with other synaptic proteins

In addition to the MAGUK family of proteins, NMDARs associate directly and indirectly with a variety of proteins localized to the PSD, including other scaffolding and signaling proteins (Husi et al., 2000). We investigated these interactions further, initially by examining the bound fraction after a single immunoprecipitation from P42 hippocampus (IP I Bound). As shown in Figure $5 A, \mathrm{nNOS}$, Homer, and $\beta$-catenin coimmunoprecipitated to a greater extent with NR2A than with NR2B. This provided the first indication that these synapse-associated proteins may interact differentially with NR2A and NR2B. Conversely, NR2A coimmu-
A

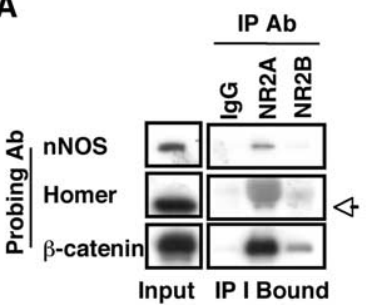

C

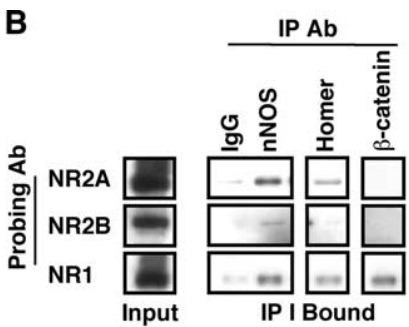

IP Ab

D

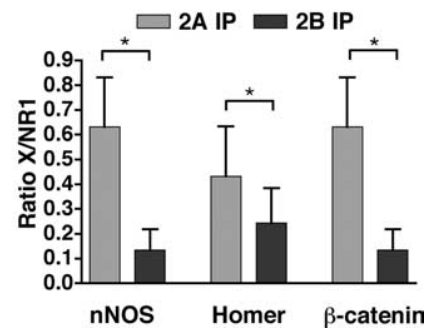

Figure 5. The synaptic proteins nNOS, Homer, and $\beta$-catenin associate to a greater extent with the di-heteromeric NR1/NR2A receptors than with NR1/NR2B receptors. $A$, Representative blots are shown of single immunoprecipitation experiments with anti-NR2A or -NR2B antibodies (Ab), probed with anti-nNOS, Homer, and $\beta$-catenin antibodies. Both NR2A and NR2B Coimmunoprecipitate the synapse-associated proteins; however, the interaction with NR2A appears to be stronger. The Homer band at $47 \mathrm{kDa}$ (noted by the arrow) may be distinguished from the higher-molecular-weight heavy chain $\lg G$ bands. $\boldsymbol{B}$, Single immunoprecipitation experiments with anti-nNOS, Homer, and $\beta$-catenin antibodies probed with anti-NR2A, NR2B, and NR1. NR1 is coimmunoprecipitated with nNOS, Homer, and $\beta$-catenin, NR2A coimmunoprecipitates with nNOS and Homer, and NR2B co-associates with nNOS, with no detectable signal in anti-Homer or anti- $\beta$-catenin immunoprecipitations. C, D, Immunoprecipitations with antiNR2A and anti-NR2B antibodies from isolated di-heteromeric receptor fractions (NR1/NR2A and NR1/NR2B) examined above were analyzed for coassociation of nNOS, Homer, and $\beta$-catenin. C, Representative blots of the IP II Unbound and IP III Bound fractions after triple immunoprecipitations and immunoblotting with anti-nNOS, - Homer, and $-\beta$-catenin antibodies. NR1 blots are identical to those in Figure 4. Densitometric analysis (D) of nNOS, Homer, or $\beta$-catenin associated with NR2A and NR2B, normalized to the level of NMDAR immunoprecipitated as measured by coimmunoprecipitated NR1. Data are representative of four (nNOS and $\beta$-catenin) or seven (Homer) independent experiments performed on different hippocampal preparations. Values are shown as mean \pm SEM. ${ }^{*} p<0.05$.

noprecipitated with nNOS and Homer, with no detectable signal in $\beta$-catenin immunoprecipitations (Fig. $5 B$ ). Although NR2B coimmunoprecipitated weakly with nNOS in these experiments, no signal was detected with Homer or $\beta$-catenin immunoprecipitations. As expected, NR1 interacted with nNOS and Homer. NR1 also coimmunoprecipitated with $\beta$-catenin (Fig. $5 B$ ), confirming the interaction of $\beta$-catenin with NMDARs seen in Figure $5 A$. Thus, NR1, but not NR2A or NR2B, coimmunoprecipitated with $\beta$-catenin, perhaps attributable to differences in antibody signals. Alternatively, if the levels of NR2A and NR2B that coimmunoprecipitated with $\beta$-catenin were below the level of detection, only when the entire NMDAR population was analyzed (by examining NR1) could the association be detected. In summary, the single immunoprecipitation experiments suggest that NR2A may associate with these proteins to a greater extent than NR2B.

Next, we analyzed the samples examined above for MAGUK associations and, using a similar approach, determined the levels of synapse-associated proteins that coimmunoprecipitate with NR1/NR2A and NR1/NR2B di-heteromeric receptors. After double immunoprecipitations with anti-NR2A or anti-NR2B antibodies, the IP II Unbound fraction was immunoprecipitated with anti-NR2B and anti-NR2A antibodies, respectively, and the IP III Bound fraction was analyzed. As shown in the representa- 
tive blot in Figure 5C, nNOS coimmunoprecipitated to a greater extent with NR2A compared with NR2B. Quantitative analysis, in which the levels of nNOS coimmunoprecipitated with NR2A or NR2B were normalized to the amount of NMDAR immunoprecipitated (as measured by NR1 coimmunoprecipitation; NR1 blots are identical to those shown in Fig. 4), revealed a significant difference between nNOS coimmunoprecipitated with NR2A and NR2B $(n=4 ; p=0.033)$ (Fig. 5D). Similarly, we investigated the interactions of the synapse-associated protein Homer with NR2A- and NR2B-containing di-heteromeric receptors. NR2A appeared to coimmunoprecipitate a larger fraction of Homer than did NR2B, as seen in the representative blot (Fig. 5C), and subsequent quantitative analysis revealed a significant difference $(n=7 ; p=0.027)$ (Fig. 5D). Finally, similar experiments revealed that $\beta$-catenin coimmunoprecipitated to a greater extent with NR2A-containing di-heteromeric receptors (Fig. 5C), and a significant difference was found after quantitative analysis $(n=4$; $p=0.026$ ) (Fig. 5D). Thus, isolated di-heteromeric NR1/NR2A receptors interact strongly with the synapse-associated proteins nNOS, Homer, and $\beta$-catenin. NR1/NR2B receptors interact to a lesser extent with the synapse-associated proteins examined.

\section{Mass spectrometry analysis reveals a novel NR2B-preferential interaction with CRMP2}

To further investigate novel interactors with NR1/NR2A or NR1/ NR2B di-heteromeric receptors, the NR2A- or NR2B-bound fractions after the triple immunoprecipitations (IP III Bound) were subjected to mass spectrometry analysis. CRMP2, a member of the collapsin response mediator protein family, was identified as a novel interactor of NR2B and was not detected in the NR2Acontaining di-heteromeric receptor fraction.

To verify the association of CRMP2 with the NMDARs, CRMP2 coimmunoprecipitation was investigated after a single immunoprecipitation with anti-NR2A or anti-NR2B antibodies from $\mathrm{P} 42$ rat hippocampus (Fig. $6 \mathrm{~A}$, IP I Bound). CRMP2 coimmunoprecipitated with both NR2A and NR2B, although a stronger signal was seen in the NR2B-associated fraction. Conversely, immunoprecipitating with an anti-CRMP2 antibody, which efficiently immunoprecipitated CRMP2 (Fig. 6B, bottom panels), revealed associations with NR1, NR2A, and NR2B. Thus, CRMP2 coimmunoprecipitates with NMDARs in $\mathrm{P} 42$ rat hippocampus.

To determine the relative associations of CRMP2 with NR2Aor NR2B-containing di-heteromeric NMDARs, P42 hippocampal membrane fractions were subjected to triple immunoprecipitations with anti-NR2A or anti-NR2B antibodies, and these fractions were analyzed for the presence of CRMP2. As seen in the representative immunoblot (Fig. 6C), CRMP2 coimmunoprecipitates to a greater extent with NR2B-containing versus NR2Acontaining di-heteromeric receptors. Quantification of the levels of CRMP2 that were coimmunoprecipitated relative to NR1, as described above, revealed a significant difference between NR2A and NR2B associations $(n=6 ; p=0.009)$ (Fig. $6 D)$. Thus, the purified NR1/NR2A or NR1/NR2B di-heteromeric receptor fraction was used to identify the novel interactor CRMP2, which favors an association with NR2B.

\section{Discussion}

We demonstrate that, throughout development, NMDARs in the hippocampus exist as three populations, i.e., NR1/NR2A, NR1/ NR2B, and NR1/NR2A/NR2B. Although NR1/NR2A and NR1/ NR2B complexes interacted similarly with PSD-95, SAP102, and PSD-93, NR1/NR2A associated to a greater extent with nNOS, Homer, and $\beta$-catenin. Mass spectrometry analysis identified a
A

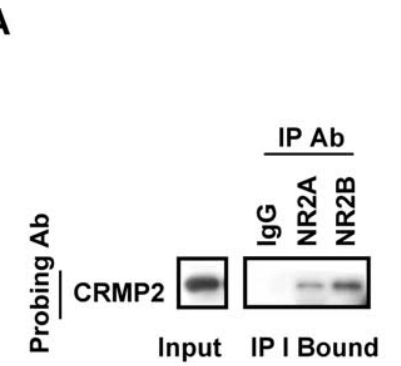

C

B
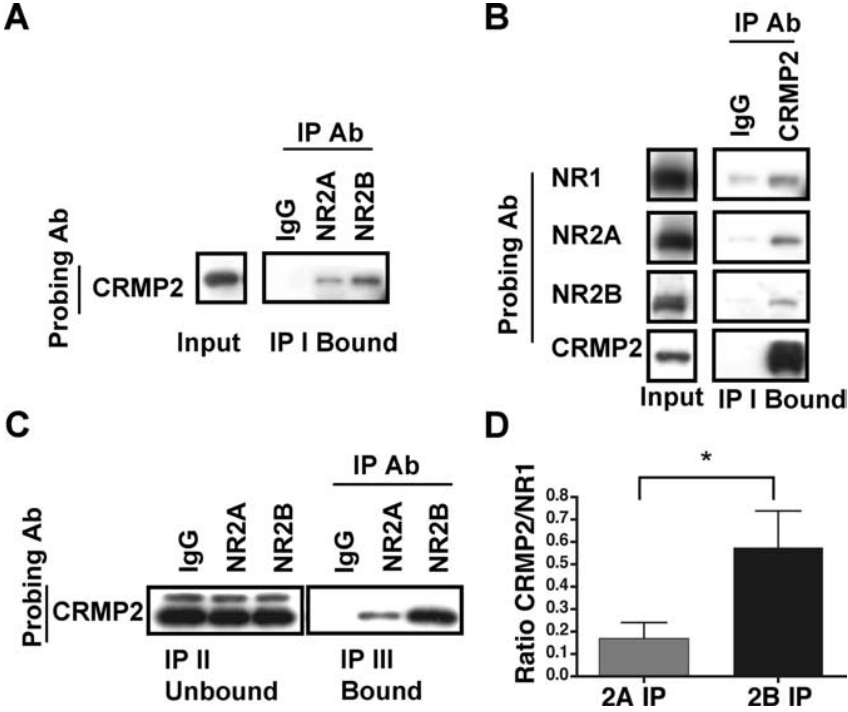

D

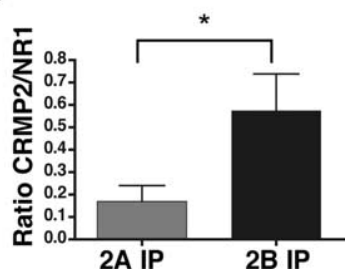

Figure 6. CRMP2 interacts preferentially with the di-heteromeric NR1/NR2B receptors. $A, A$ representative blot of the input and IP I Bound fractions after immunoprecipitations with antiNR2A and -NR2B antibodies (Ab). Immunoblot analysis with an anti-CRMP2 antibody demonstrated that CRMP2 coimmunoprecipitates with NR2A and NR2B from P42 hippocampus. $\boldsymbol{B}$, Representative blots of the input and IP I Bound fractions after immunoprecipitations with an anti-CRMP2 antibody. Immunoblot analysis with anti-NR1, -NR2A, or -NR2B antibodies demonstrated that CRMP2 coimmunoprecipitates the NMDAR subunits from P42 hippocampus. $C$ Immunoprecipitations with anti-NR2A and anti-NR2B antibodies from isolated di-heteromeric receptor fractions were analyzed for coassociation of CRMP2. A representative blot of the IP II Unbound and IP III Bound fractions after immunoblotting with anti-CRMP2 antibodies. CRMP2 associates to a greater extent with the NR2B subunit. D, Densitometric analysis of CRMP2 Coimmunoprecipitated with NR2A and NR2B, normalized to the level of NMDAR immunoprecipitated as measured by coimmunoprecipitated NR1. Data are representative of six independent experiments performed on different CA1/CA2 preparations. Values are shown as mean $\pm S E M$. ${ }^{*} p<0.05$.

novel interactor, CRMP2, which associated to a greater extent with NR1/NR2B receptors. Thus, sizeable populations of diheteromeric NMDARs are preferentially localized in separate protein complexes, despite no measurable difference in the levels of associated MAGUKs.

The initial step in the serial immunoprecipitation protocol involves extraction of NMDARs from membrane fractions. Previous studies have reported that NMDARs are relatively insoluble in Triton X-100, but 35\% of NR2A and NR2B subunits are extracted in 1\% DOC (Blahos and Wenthold, 1996). In our study, DOC solubilization yielded similar levels of extracted NMDAR subunits in P42 and 6-month-old CA1/CA2 as reported previously for adult forebrain. Surprisingly, nearly all NR2A, NR2B, and NR1 subunits were extracted from $\mathrm{P} 7$ rat hippocampus. This significantly higher level of solubility at P7 suggests that either the mechanisms responsible for tethering NMDARs are not fully developed until later in synapse development or NMDARs are localized extrasynaptically or intracellularly. Proposed mechanisms of NMDAR anchoring include cytoskeletal interactions, which are necessary for NMDAR synaptic localization but not clustering (Allison et al., 1998), binding to PSD scaffolding proteins independently of cytoskeletal interactions (Allison et al., 2000), and localization to Triton X-100-insoluble lipid rafts (Suzuki et al., 2001; Hering et al., 2003). Although a small number of developed PSD structures have been reported as early as P2 (Petralia et al., 2005), NMDARs in primary cortical neurons at $4 \mathrm{~d}$ in vitro are primarily extrasynaptic as they cycle in and out of dendritic plasma membranes before synapse formation (Wash- 
bourne et al., 2004). Thus, increased solubility at P7 may be a result of extrasynaptic or intracellular NMDAR localization. However, immunogold analysis has demonstrated that NMDARs are present at similar levels in rat CA1 synapses at P2, P10, and adult (Petralia et al., 1999). Therefore, the increased solubility of synaptic NMDAR subunits at P7 may be attributable to looser associations of the NMDARs with immature PSDs.

Because of relatively low levels of DOC solubility in older animals, NMDARs examined by serial immunoprecipitations may not represent all NMDAR subpopulations in the neuron. For example, intracellular or extrasynaptic receptors may be more easily extracted, excluding synaptic populations from analysis. If this were the case, analysis of a more soluble sample would potentially reveal different results; however, the proportion of di-heteromeric receptors at P7 was similar to that at P42 and 6 months, despite threefold higher subunit solubility at P7. Furthermore, our subsequent experiments investigating associations with synapse-associated proteins revealed robust interactions, suggesting that synaptic NMDARs were extracted by DOC solubilization. We cannot rule out the possibility that some of the solubilized NMDARs represent intracellular receptors associated with endosomes or transport vesicles. These technical limitations are shared among biochemical studies of NMDARs.

We report a similar proportion of NR1/NR2A and NR1/NR2B di-heteromeric receptors at P7, P42, and 6 months, despite changes in the expression of NR2A in relation to NR2B. The ratio of NR2A to NR2B is low early in development but increases as NR2A expression increases and NR2B expression decreases (Watanabe et al., 1993; Wenzel et al., 1997; Sans et al., 2000). Changes in relative subunit expression could potentially be reflected in NMDAR populations; however, the relative proportion of diheteromeric NMDARs did not change significantly over development. Surprisingly, despite relatively low expression of NR2A at P7, a large fraction of NR2A was not bound to NR2B, suggesting that the formation of di-heteromeric receptors may be preferred. Thus, subunit composition is not simply a result of the random assembly of available NR2A and NR2B subunits but may be regulated by an undetermined mechanism, such as chaperones that facilitate the formation of preferred complexes or differences in affinities of subunits for one another, as suggested for AMPA receptors (Sans et al., 2003). Notably, our analysis of NMDAR complexes at P7 may be affected by the presence of the NR2D subunit, which is expressed in hippocampal cells during early development (Monyer et al., 1994; Kirson et al., 1999) or by hippocampal cells outside of CA1/CA2.

At the ages examined, $\sim 60-85 \%$ of NR2A and NR2B did not coimmunoprecipitate with one another. The remaining 15-40\% of NR2A and NR2B that were associated likely represent the triheteromeric receptor population. Tri-heteromeric receptors have been reported by electrophysiological (Tovar and Westbrook, 1999) and coimmunoprecipitation (Sheng et al., 1994; Blahos and Wenthold, 1996) studies of NR2A and NR2B; however, the relative size of this population in the hippocampus had not previously been investigated. In contrast to studies in cortex and striatum, in which more than half of NMDARs contain both NR2A and NR2B (Luo et al., 1997; Dunah and Standaert, 2003), our results indicate that the tri-heteromeric receptor population constitutes one-third of the total receptor population. Although our studies do not directly compare NMDAR subunit composition across brain regions, these data support a regulated mechanism of subunit assembly because NMDAR subunit composition differs across regions in which NR2A and NR2B are the predom- inant NR2 subunits, such as hippocampus and cortex (Wenzel et al., 1997).

Previous studies have suggested that the NMDAR interacts differentially with MAGUKs in an NR2-dependent manner with a correlation between NR2A and PSD-95 and between NR2B and SAP102 in hippocampus and visual cortex (Sans et al., 2000; Townsend et al., 2003). Our current results indicate, however, that the isolated di-heteromeric receptor fractions do not interact differentially with PSD-95, SAP102, or PSD-93. Additionally, MAGUKs are modified posttranslationally (Topinka and Bredt, 1998; Craven and Bredt, 2000; Morabito et al., 2004). Although the possibility remains that the MAGUKs associated with NR2Aor NR2B-containing receptors may differ with respect to these modifications, the distinct NR1/NR2A and NR1/NR2B complexes are not defined by differences in the abundance of associated MAGUKs.

NMDARs have been shown previously to coimmunoprecipitate with nNOS (Christopherson et al., 1999), Homer (Husi et al., 2000), and $\beta$-catenin (Husi et al., 2000). We demonstrate for the first time the NR2A-dominant nature of these interactions. These findings are consistent with the model that synaptic NMDARs are NR2A rich and extrasynaptic receptors are NR2B rich in adult hippocampus (Tovar and Westbrook, 1999; Thomas et al., 2006). The association of nNOS, Homer, and $\beta$-catenin was not exclusive for NR2A, i.e., these proteins coimmunoprecipitated with $\mathrm{NR} 2 \mathrm{~B}$, consistent with previous reports that the synaptic and extrasynaptic segregation of NR2A and NR2B, respectively, is not exclusive (Kohr, 2006; Thomas et al., 2006).

No evidence exists for direct interactions between NMDARs and the synapse-associated proteins we examined, suggesting indirect associations through intermediate binding proteins. PSD-95 is necessary for the association of the nNOS/NMDAR complex (Christopherson et al., 1999); Homer interaction with NMDARs is mediated by Shank/guanylate kinase domainassociated protein/PSD-95 (Tu et al., 1999). Although $\beta$-catenin association with NMDARs or the PSD-95 family of proteins has not been directly investigated, $\beta$-catenin contains a PDZ binding domain (Perego et al., 2000) and associates with the MAGUK protein synaptic scaffolding molecule (S-SCAM or membraneassociated guanylate kinase inverted-1) (Dobrosotskaya and James, 2000; Nishimura et al., 2002). S-SCAM interacts with NMDARs (Hirao et al., 1998) and the GK domain of PSD-95 (Hirao et al., 2000), providing two mechanisms by which $\beta$-catenin may be linked to NMDARs (i.e., $\beta$-catenin/S-SCAM/ NMDARs or $\beta$-catenin/S-SCAM/PSD-95/NMDARs). Interestingly, although we found no preferential interaction of NR2A or NR2B with the MAGUKs, which are directly associated with NMDARs (Niethammer et al., 1996) and represent first-order interacting proteins, we identified differences in higher-order associations with nNOS, Homer, and $\beta$-catenin. Notably, this suggests that the same MAGUKs may interact differentially with these higher-order proteins, consistent with a previous study that used a similar serial immunoprecipitation protocol and identified a preferential interaction of synaptic ras-GTPase-activating protein with NR2B-containing NMDARs, although both NR2A and NR2B coimmunoprecipitated PSD-95 (Kim et al., 2005).

Mass spectrometry analysis of the isolated di-heteromeric receptors identified CRMP2 as a preferential interactor of NR2B. CRMP2 is a microtubule-associated protein previously implicated in regulating NR2B surface expression in response to NMDAR-dependent calpain cleavage (Bretin et al., 2006). CRMP2 interacts with tubulin dimers (Fukata et al., 2002; Arimura et al., 2005) in a phosphorylation-dependent manner 
(Arimura et al., 2005) and is involved in the internalization of the L1 receptor from the cell surface (Nishimura et al., 2003), suggesting a potential role for CRMP2 in trafficking events. Also, mice lacking the CRMP family member CRMP1 exhibit impaired LTP and spatial learning and memory (Su et al., 2007). Thus, this interaction warrants additional studies to elucidate the function of CRMP2 in NMDAR trafficking or regulation.

In this study, we demonstrate that the NMDAR exists as three populations throughout development, and the sizeable NR1/ NR2A and NR1/NR2B di-heteromeric receptor populations are localized to different protein complexes in adult hippocampus. These findings support previous proposals of NR2A- or NR2Bspecific functions and provide a structure that may dictate these differential contributions.

\section{References}

Al-Hallaq RA, Jarabek BR, Fu Z, Vicini S, Wolfe BB, Yasuda RP (2002) Association of NR3A with the N-methyl-D-aspartate receptor NR1 and NR2 subunits. Mol Pharmacol 62:1119-1127.

Allison DW, Gelfand VI, Spector I, Craig AM (1998) Role of actin in anchoring postsynaptic receptors in cultured hippocampal neurons: differential attachment of NMDA versus AMPA receptors. J Neurosci 18:2423-2436.

Allison DW, Chervin AS, Gelfand VI, Craig AM (2000) Postsynaptic scaffolds of excitatory and inhibitory synapses in hippocampal neurons: maintenance of core components independent of actin filaments and microtubules. J Neurosci 20:4545-4554.

Arimura N, Menager C, Kawano Y, Yoshimura T, Kawabata S, Hattori A, Fukata Y, Amano M, Goshima Y, Inagaki M, Morone N, Usukura J, Kaibuchi K (2005) Phosphorylation by Rho kinase regulates CRMP-2 activity in growth cones. Mol Cell Biol 25:9973-9984.

Besshoh S, Bawa D, Teves L, Wallace MC, Gurd JW (2005) Increased phosphorylation and redistribution of NMDA receptors between synaptic lipid rafts and post-synaptic densities following transient global ischemia in the rat brain. J Neurochem 93:186-194.

Blahos II J, Wenthold RJ (1996) Relationship between N-methyl-Daspartate receptor NR1 splice variants and NR2 subunits. J Biol Chem 271:15669-15674.

Bretin S, Rogemond V, Marin P, Maus M, Torrens Y, Honnorat J, Glowinski J, Premont J, Gauchy C (2006) Calpain product of WT-CRMP2 reduces the amount of surface NR2B NMDA receptor subunit. J Neurochem 98:1252-1265.

Chazot PL, Stephenson FA (1997) Biochemical evidence for the existence of a pool of unassembled C2 exon-containing NR1 subunits of the mammalian forebrain NMDA receptor. J Neurochem 68:507-516.

Christie JM, Wenthold RJ, Monaghan DT (1999) Insulin causes a transient tyrosine phosphorylation of NR2A and NR2B NMDA receptor subunits in rat hippocampus. J Neurochem 72:1523-1528.

Christopherson KS, Hillier BJ, Lim WA, Bredt DS (1999) PSD-95 assembles a ternary complex with the $N$-methyl-D-aspartic acid receptor and a bivalent neuronal NO synthase PDZ domain. J Biol Chem 274:27467-27473.

Craven SE, Bredt DS (2000) Synaptic targeting of the postsynaptic density protein PSD-95 mediated by a tyrosine-based trafficking signal. J Biol Chem 275:20045-20051.

Cull-Candy S, Brickley S, Farrant M (2001) NMDA receptor subunits: diversity, development and disease. Curr Opin Neurobiol 11:327-335.

Cull-Candy SG, Leszkiewicz DN (2004) Role of distinct NMDA receptor subtypes at central synapses. Sci STKE 2004:re16.

Dobrosotskaya IY, James GL (2000) MAGI-1 interacts with beta-catenin and is associated with cell-cell adhesion structures. Biochem Biophys Res Commun 270:903-909.

Dunah AW, Standaert DG (2003) Subcellular segregation of distinct heteromeric NMDA glutamate receptors in the striatum. J Neurochem 85:935-943.

Fukata Y, Itoh TJ, Kimura T, Menager C, Nishimura T, Shiromizu T, Watanabe $\mathrm{H}$, Inagaki $\mathrm{N}$, Iwamatsu $\mathrm{A}$, Hotani $\mathrm{H}$, Kaibuchi $\mathrm{K}$ (2002) CRMP-2 binds to tubulin heterodimers to promote microtubule assembly. Nat Cell Biol 4:583-591.

Furukawa H, Singh SK, Mancusso R, Gouaux E (2005) Subunit arrangement and function in NMDA receptors. Nature 438:185-192.
Gardoni F, Di Luca M (2006) New targets for pharmacological intervention in the glutamatergic synapse. Eur J Pharmacol 545:2-10.

Gardoni F, Mauceri D, Fiorentini C, Bellone C, Missale C, Cattabeni F, Di Luca M (2003) CaMKII-dependent phosphorylation regulates SAP97/ NR2A interaction. J Biol Chem 278:44745-44752.

Hering H, Lin CC, Sheng M (2003) Lipid rafts in the maintenance of synapses, dendritic spines, and surface AMPA receptor stability. J Neurosci 23:3262-3271.

Hirao K, Hata Y, Ide N, Takeuchi M, Irie M, Yao I, Deguchi M, Toyoda A, Sudhof TC, Takai Y (1998) A novel multiple PDZ domain-containing molecule interacting with $N$-methyl-D-aspartate receptors and neuronal cell adhesion proteins. J Biol Chem 273:21105-21110.

Hirao K, Hata Y, Yao I, Deguchi M, Kawabe H, Mizoguchi A, Takai Y (2000) Three isoforms of synaptic scaffolding molecule and their characterization. Multimerization between the isoforms and their interaction with $\mathrm{N}$-methyl-D-aspartate receptors and SAP90/PSD-95-associated protein. J Biol Chem 275:2966-2972.

Huh KH, Wenthold RJ (1999) Turnover analysis of glutamate receptors identifies a rapidly degraded pool of the $\mathrm{N}$-methyl-D-aspartate receptor subunit, NR1, in cultured cerebellar granule cells. J Biol Chem 274:151-157.

Husi H, Ward MA, Choudhary JS, Blackstock WP, Grant SG (2000) Proteomic analysis of NMDA receptor-adhesion protein signaling complexes. Nat Neurosci 3:661-669.

Kennedy MB, Manzerra P (2001) Telling tails. Proc Natl Acad Sci USA 98:12323-12324.

Kew JN, Richards JG, Mutel V, Kemp JA (1998) Developmental changes in NMDA receptor glycine affinity and ifenprodil sensitivity reveal three distinct populations of NMDA receptors in individual rat cortical neurons. J Neurosci 18:1935-1943.

Kim MJ, Dunah AW, Wang YT, Sheng M (2005) Differential roles of NR2Aand NR2B-containing NMDA receptors in Ras-ERK signaling and AMPA receptor trafficking. Neuron 46:745-760.

Kirson ED, Schirra C, Konnerth A, Yaari Y (1999) Early postnatal switch in magnesium sensitivity of NMDA receptors in rat CA1 pyramidal cells. J Physiol (Lond) 521:99-111.

Kohr G (2006) NMDA receptor function: subunit composition versus spatial distribution. Cell Tissue Res 326:439-446.

Lin Y, Skeberdis VA, Francesconi A, Bennett MV, Zukin RS (2004) Postsynaptic density protein-95 regulates NMDA channel gating and surface expression. J Neurosci 24:10138-10148.

Losi G, Prybylowski K, Fu Z, Luo J, Wenthold RJ, Vicini S (2003) PSD-95 regulates NMDA receptors in developing cerebellar granule neurons of the rat. J Physiol (Lond) 548:21-29.

Luo J, Wang Y, Yasuda RP, Dunah AW, Wolfe BB (1997) The majority of $\mathrm{N}$-methyl-D-aspartate receptor complexes in adult rat cerebral cortex contain at least three different subunits (NR1/NR2A/NR2B). Mol Pharmacol 51:79-86.

Lynch DR, Guttmann RP (2002) Excitotoxicity: perspectives based on N-methyl-D-aspartate receptor subtypes. J Pharmacol Exp Ther 300:717-723.

Lynch MA (2004) Long-term potentiation and memory. Physiol Rev 84:87-136.

McBain CJ, Mayer ML (1994) N-methyl-D-aspartic acid receptor structure and function. Physiol Rev 74:723-760.

Monyer H, Burnashev N, Laurie DJ, Sakmann B, Seeburg PH (1994) Developmental and regional expression in the rat brain and functional properties of four NMDA receptors. Neuron 12:529-540.

Morabito MA, Sheng M, Tsai LH (2004) Cyclin-dependent kinase 5 phosphorylates the $\mathrm{N}$-terminal domain of the postsynaptic density protein PSD-95 in neurons. J Neurosci 24:865-876.

Niethammer M, Kim E, Sheng M (1996) Interaction between the C terminus of NMDA receptor subunits and multiple members of the PSD-95 family of membrane-associated guanylate kinases. J Neurosci 16:2157-2163.

Nishimura T, Fukata Y, Kato K, Yamaguchi T, Matsuura Y, Kamiguchi H, Kaibuchi K (2003) CRMP-2 regulates polarized Numb-mediated endocytosis for axon growth. Nat Cell Biol 5:819-826.

Nishimura W, Yao I, Iida J, Tanaka N, Hata Y (2002) Interaction of synaptic scaffolding molecule and $\beta$-catenin. J Neurosci 22:757-765.

Perego C, Vanoni C, Massari S, Longhi R, Pietrini G (2000) Mammalian 
LIN-7 PDZ proteins associate with beta-catenin at the cell-cell junctions of epithelia and neurons. EMBO J 19:3978-3989.

Petralia RS, Esteban JA, Wang YX, Partridge JG, Zhao HM, Wenthold RJ, Malinow R (1999) Selective acquisition of AMPA receptors over postnatal development suggests a molecular basis for silent synapses. Nat Neurosci 2:31-36.

Petralia RS, Sans N, Wang YX, Wenthold RJ (2005) Ontogeny of postsynaptic density proteins at glutamatergic synapses. Mol Cell Neurosci 29:436-452.

Prybylowski K, Chang K, Sans N, Kan L, Vicini S, Wenthold RJ (2005) The synaptic localization of NR2B-containing NMDA receptors is controlled by interactions with PDZ proteins and AP-2. Neuron 47:845-857.

Roche KW, Standley S, McCallum J, Dune Ly C, Ehlers MD, Wenthold RJ (2001) Molecular determinants of NMDA receptor internalization. Nat Neurosci 4:794-802.

Salter MW, Kalia LV (2004) Src kinases: a hub for NMDA receptor regulation. Nat Rev Neurosci 5:317-328.

Sans N, Petralia RS, Wang YX, Blahos J, 2nd, Hell JW, Wenthold RJ (2000) A developmental change in NMDA receptor-associated proteins at hippocampal synapses. J Neurosci 20:1260-1271.

Sans N, Vissel B, Petralia RS, Wang YX, Chang K, Royle GA, Wang CY, O'Gorman S, Heinemann SF, Wenthold RJ (2003) Aberrant formation of glutamate receptor complexes in hippocampal neurons of mice lacking the GluR2 AMPA receptor subunit. J Neurosci 23:9367-9373.

Sheng M, Cummings J, Roldan LA, Jan YN, Jan LY (1994) Changing subunit composition of heteromeric NMDA receptors during development of rat cortex. Nature 368:144-147.

Stephenson FA (2001) Subunit characterization of NMDA receptors. Curr Drug Targets 2:233-239.

Stocca G, Vicini S (1998) Increased contribution of NR2A subunit to synaptic NMDA receptors in developing rat cortical neurons. J Physiol (Lond) 507:13-24.

Su KY, Chien WL, Fu WM, Yu IS, Huang HP, Huang PH, Lin SR, Shih JY, Lin YL, Hsueh YP, Yang PC, Lin SW (2007) Mice deficient in collapsin response mediator protein-1 exhibit impaired long-term potentiation and impaired spatial learning and memory. J Neurosci 27:2513-2524.

Suzuki T, Ito J, Takagi H, Saitoh F, Nawa H, Shimizu H (2001) Biochemical evidence for localization of AMPA-type glutamate receptor subunits in the dendritic raft. Brain Res Mol Brain Res 89:20-28.

Thomas CG, Miller AJ, Westbrook GL (2006) Synaptic and extrasynaptic
NMDA receptor NR2 subunits in cultured hippocampal neurons. J Neurophysiol 95:1727-1734.

Topinka JR, Bredt DS (1998) N-terminal palmitoylation of PSD-95 regulates association with cell membranes and interaction with $\mathrm{K}^{+}$channel Kv1.4. Neuron 20:125-134.

Tovar KR, Westbrook GL (1999) The incorporation of NMDA receptors with a distinct subunit composition at nascent hippocampal synapses in vitro. J Neurosci 19:4180-4188.

Townsend M, Yoshii A, Mishina M, Constantine-Paton M (2003) Developmental loss of miniature $\mathrm{N}$-methyl-D-aspartate receptor currents in NR2A knockout mice. Proc Natl Acad Sci USA 100:1340-1345.

Tu JC, Xiao B, Naisbitt S, Yuan JP, Petralia RS, Brakeman P, Doan A, Aakalu VK, Lanahan AA, Sheng M, Worley PF (1999) Coupling of mGluR/ Homer and PSD-95 complexes by the Shank family of postsynaptic density proteins. Neuron 23:583-592.

Vanhoutte P, Bading H (2003) Opposing roles of synaptic and extrasynaptic NMDA receptors in neuronal calcium signalling and BDNF gene regulation. Curr Opin Neurobiol 13:366-371.

Wafford KA, Bain CJ, Le Bourdelles B, Whiting PJ, Kemp JA (1993) Preferential co-assembly of recombinant NMDA receptors composed of three different subunits. NeuroReport 4:1347-1349.

Wang YH, Bosy TZ, Yasuda RP, Grayson DR, Vicini S, Pizzorusso T, Wolfe BB (1995) Characterization of NMDA receptor subunit-specific antibodies: distribution of NR2A and NR2B receptor subunits in rat brain and ontogenic profile in the cerebellum. J Neurochem 65:176-183.

Washbourne P, Liu XB, Jones EG, McAllister AK (2004) Cycling of NMDA receptors during trafficking in neurons before synapse formation. J Neurosci 24:8253-8264.

Watanabe M, Inoue Y, Sakimura K, Mishina M (1993) Distinct distributions of five $\mathrm{N}$-methyl-D-aspartate receptor channel subunit mRNAs in the forebrain. J Comp Neurol 338:377-390.

Wenthold RJ, Prybylowski K, Standley S, Sans N, Petralia RS (2003) Trafficking of NMDA receptors. Annu Rev Pharmacol Toxicol 43:335-358.

Wenzel A, Fritschy JM, Mohler H, Benke D (1997) NMDA receptor heterogeneity during postnatal development of the rat brain: differential expression of the NR2A, NR2B, and NR2C subunit proteins. J Neurochem 68:469-478.

Wilm M, Shevchenko A, Houthaeve T, Breit S, Schweigerer L, Fotsis T, Mann M (1996) Femtomole sequencing of proteins from polyacrylamide gels by nano-electrospray mass spectrometry. Nature 379:466-469. 